\title{
Journal of Neuro Oncology: immunotherapy for brain tumors
}

\author{
Maryam Rahman ${ }^{1} \cdot$ Ian Parney ${ }^{2}$ \\ Published online: 20 October 2020 \\ (c) Springer Science+Business Media, LLC, part of Springer Nature 2020
}

As we write this editorial, the world has stood up against systematic and institutionalized racism that has resulted in the premature death and subjugation of countless Black men and women in the United States. During these times, scientific discussions about brain tumors and therapeutic discovery seems even more difficult and the call for a cure even further from reach. We have created this special issue in collaboration with successful and diverse scientists and clinicians throughout the country and feel the content is novel and provides a basis for the future of brain tumor immunotherapy. As you read these articles and listen to the webinars created for this effort, please know that we all join together with our global community in protest against the violent and non-violent measures used to prevent Black Americans and other under-represented minorities from living full and healthy lives that are free from fear. Our efforts to bring this work to fruition is not due to lack of activism for our brothers and sisters.

Immunotherapy for cancer has revolutionized the care for certain solid tissue malignancies resulting in durable survival benefit for patients with traditionally treatment resistant cancers like melanoma and renal cell carcinoma. This enthusiasm has led to vigorous research efforts using various immunotherapeutic platforms for brain metastases and malignant glioma. These efforts have been thoughtful with well-designed clinical trials that have informed future steps. Based on this work we now understand the profound immunosuppression associated with intracranial malignancies, the role of immune escape and the potential utility of combinatorial strategies to increase immune responses. The future of brain tumor treatment will rely on our ability to successfully identify which patients will benefit from what therapy and how to evaluate treatment response prior to

Maryam Rahman

Maryam.Rahman@neurosurgery.ufl.edu

Ian Parney

parney.ian@mayo.edu

1 University of Florida, Gainesville, FL, USA

2 Mayo Clinic, Rochester, MN, USA tumor progression. Immunotherapy lends itself nicely to this paradigm by leveraging host immunity, providing the ability to target diverse antigens and the ability to measure immune responses prior to determining tumor responses on imaging.

This special issue is designed to present current and future research in immunotherapy for brain tumors and provide didactic modules on immunity and immunotherapy through free webinars available on the AANS/CNS Joint Tumor Section website. The issue begins with comprehensive articles by Dr. Fecci's and Dr. Gilbert's groups discussing host and iatrogenic factors associated with immunosuppression in glioblastoma. This is followed by discussion by Dr. Flores, Dr. Sayour, Dr. Lim, Dr. Okada and Dr. Sampson on novel approaches to brain tumor immunotherapy. The special issue concludes with methods to combine immunotherapy with ablation techniques from Dr. Heimberger, Dr. Sheehan and Dr. Tran. This special issue reflects the important work that has been performed for the last five years and the beginning of the work that will ensue for the next five years to establish the role of immunotherapy for the treatment of brain tumors. There is much work to do.

Far and away the best prize that life offers is the chance to work hard at work worth doing.

Theodore Roosevelt, Sept 7th 1903.

For free educational webinars on relevant immunotherapy topics (hosted by the AANS/CNS Section on Tumors at https ://www.tumorsection.org/), please access:

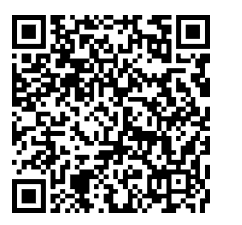

Publisher's Note Springer Nature remains neutral with regard to jurisdictional claims in published maps and institutional affiliations. 